\title{
MDM2 SNP309 and SNP285 Act as Negative Prognostic Markers for Non-small Cell Lung Cancer Adenocarci- noma Patients
}

\author{
Christophe Deben ${ }^{1,2}{ }^{\varpi}$, Ken Op de Beeck ${ }^{1,4}$, Jolien Van den Bossche ${ }^{1}$, Julie Jacobs ${ }^{1,2}$, Filip Lardon ${ }^{1}$, An Wouters ${ }^{1}$, \\ Marc Peeters ${ }^{1,3}$, Guy Van Camp, ${ }^{1,}$, Christian Rolfo ${ }^{3,5}$, Vanessa Deschoolmeester ${ }^{1,2 *}$ and Patrick Pauwels ${ }^{1,2 *}$ \\ 1. Center for Oncological Research (CORE), University of Antwerp, Universiteitsplein 1, 2610 Wilrijk, Antwerp, Belgium; \\ 2. Department of Pathology, Antwerp University Hospital, Wilrijkstraat 10, 2650 Edegem, Antwerp, Belgium; \\ 3. Department of Medical Oncology, Antwerp University Hospital, Wilrijkstraat 10, 2650 Edegem, Antwerp, Belgium; \\ 4. Center of Medical Genetics, University of Antwerp \& Antwerp University Hospital, Wilrijkstraat 10, 2650 Edegem, Antwerp, Belgium; \\ 5. Phase-1 Early Clinical Trials Unit, Antwerp University Hospital Wilrijkstraat 10, 2650 Edegem, Antwerp, Belgium. \\ * These authors are co-senior authors \\ $\triangle$ Corresponding author: Christophe Deben, christophe.deben@uantwerpen.be; +3232652533; Center for Oncological Research, Antwerp University, \\ Universiteitsplein 1 T4.35, 2610 Wilrijk, Antwerp, Belgium. \\ (c) Ivyspring International Publisher. This is an open access article distributed under the terms of the Creative Commons Attribution (CC BY-NC) license \\ (https:// creativecommons.org/licenses/by-nc/4.0/). See http://ivyspring.com/terms for full terms and conditions.
}

Received: 2017.01.19; Accepted: 2017.04.26; Published: 2017.07.15

\begin{abstract}
Objectives: Two functional polymorphisms in the MDM2 promoter region, SNP309T>G and SNP285G>C, have been shown to impact MDM2 expression and cancer risk. Currently available data on the prognostic value of MDM2 SNP309 in non-small cell lung cancer (NSCLC) is contradictory and unavailable for SNP285. The goal of this study was to clarify the role of these MDM2 SNPs in the outcome of NSCLC patients.

Materials and Methods: In this study we genotyped SNP309 and SNP285 in 98 NSCLC adenocarcinoma patients and determined MDM2 mRNA and protein levels. In addition, we assessed the prognostic value of these common SNPs on overall and progression free survival, taking into account the TP53 status of the tumor.

Results and Conclusion: We found that the SNP285C allele, but not the SNP309G allele, was significantly associated with increased MDM2 mRNA expression levels $(p=0.025)$. However, we did not observe an association with MDM2 protein levels for SNP285. The SNP309G allele was significantly associated with the presence of wild type TP53 $(p=0.047)$ and showed a strong trend towards increased MDM2 protein levels $(p$ $=0.068$ ). In addition, patients harboring the SNP309G allele showed a worse overall survival, but only in the presence of wild type TP53. The SNP285C allele was significantly associated with an early age of diagnosis and metastasis. Additionally, the SNP285C allele acted as an independent predictor for worse progression free survival $(\mathrm{HR}=3.97 ; 95 \% \mathrm{Cl}=1.51-10.42 ; \mathrm{p}=0.005)$.

Our data showed that both SNP309 (in the presence of wild type TP53) and SNP285 act as negative prognostic markers for NSCLC patients, implicating a prominent role for these variants in the outcome of these patients.
\end{abstract}

Key words: MDM2, SNP309, SNP285, NSCLC, Prognostic.

\section{Introduction}

The murine double minute 2 (MDM2) protein is the principal cellular regulator of the p53 protein through a tightly regulated negative feedback loop [1]. MDM2 is the product of a p53 inducible gene and acts as an E3-ubiquitin ligase for p53, thereby targeting the protein for proteasomal degradation. In addition, MDM2 is able to bind p53, leading to the inhibition of its transcriptional activity and to its translocation from the nucleus into the cytoplasm [2].

MDM2 can exert its role as an oncoprotein through overexpression induced by MDM2 gene amplification or through the presence of a single nucleotide polymorphism (SNP309T>G, rs2279744) located within the MDM2 P2 promoter region. The 
SNP309G allele is found in approximately $37 \%$ of the population and has been shown to increase the binding affinity for the SP1 (specificity protein 1) transcription factor, resulting in increased MDM2 expression and thereby reducing wild type p53 levels and blocking p53's transcriptional activity [3]. As reviewed previously, the role of SNP309 has been studied extensively in the context of cancer risk, resulting in conflicting data, which can partially be explained by gender and ethnical differences [4].

More recently, Knappskog et al. reported a second polymorphism (SNP285G>C; rs117039649) in the same MDM2 promoter region, situated $24 \mathrm{bps}$ upstream of SNP309 [5]. The SNP285C allele has a lower frequency than SNP309G allele and is primarily observed in Caucasians [5, 6]. The SNP285C allele is in linkage disequilibrium with the SNP309G allele, resulting in the lack of the SNP309T/SNP285C haplotype $[7,8]$. The SNP285C allele seems to antagonize the effect of the SNP309G allele by reducing the binding affinity of the SP1 transcription factor to the MDM2 promoter region [5]. Again, the presence of this SNP is mostly studied for its role in cancer risk and has been associated with a reduced risk of ovarian, breast and endometrial cancers, but was not associated with risk of prostate or lung cancer, thus suggesting a gender specific protective role, possibly related to the presence of estrogen [8-10].

In this study, we focused on the prognostic role of SNP309 and SNP285 in a Caucasian (Belgian) patient population diagnosed with non-small cell lung cancer adenocarcinoma (NSCLC). Currently, only a limited number of studies have focused on the prognostic role of the SNP309G allele which resulted in highly conflicting results, as discussed previously by our group [4]. This indicates the need for further studies to determine the true prognostic role of SNP309 in NSCLC. To our knowledge, the prognostic value of SNP285 has not been studied previously in NSCLC and will therefore be explored in this study. In addition, we determined the association of SNP309 or SNP285 with MDM2 transcription and translation by defining MDM2 mRNA and protein levels. Finally, the patients' p53 status was determined and taken into account as a factor possibly influencing the prognostic value of MDM2 SNP309, which other studies failed to include.

\section{Materials and Methods}

\section{Patients}

A total of 98 NSCLC patients were selected by an independent researcher based on histological subtype (adenocarcinoma). Tissue samples from tumor resections were obtained from the Antwerp University Hospital Tumorbank, which is funded by the National Cancer Plan. Tissue specimens were fixed in $4 \%$ formaldehyde for $6-18 \mathrm{~h}$ and paraffin embedded on a routine basis. An additional 4 FFPE specimens from NSCLC patients, without malignant cells, constituted the control samples. Patients were diagnosed between 2005 and 2014, therefore EGFR/ALK status was available for a limited number of patients and not taken into account.

\section{DNA and RNA extraction and Quantification}

DNA and RNA were extracted from 5-10 FFPE tissue slides (10 $\mu \mathrm{m}$ thick), depending on the size of the tumor region. Tumor regions were determined by an experienced pathologist based on hematoxylin and eosin stained reference slides and enriched by macrodissection. DNA was isolated using the QIAmp DNA FFPE tissue kit (Qiagen, Venlo, the Netherlands), according to the manufacturer's instructions. RNA was isolated using the RNeasy FFPE isolation kit \#74404 (Qiagen). DNA and RNA concentrations were measured on a Qubit 2.0 fluorometer using the Qubit dsDNA BR assay and Qubit RNA BR assay (Invitrogen, Merelbeke, Belgium), respectively, as previously described [11]. The purity (A260/280 ratio and A260/230 ratio) was measured using the NanoDrop ND-1000 spectrophotometer (ThermoFisher Scientific, Gent, Belgium).

\section{MDM2 SNP309/SNP285 genotyping}

Genotyping of the MDM2 SNP309T/G and SNP285G/C polymorphism was performed on 98 genomic tumor DNA samples and 4 normal lung tissue samples by high resolution melting analysis. PCR $\left[1 \times\left(95^{\circ} \mathrm{C} / 10 \mathrm{sec}\right) ; 40 \times\left(95^{\circ} \mathrm{C} / 10 \mathrm{sec}, 62^{\circ} \mathrm{C} / 10 \mathrm{sec}\right.\right.$, $72^{\circ} \mathrm{C} / 10$ sec $) ; 1 \times\left(95^{\circ} \mathrm{C} / 30\right.$ sec $\left.)\right]$ was performed with a Lightscanner master mix with the addition of $0.6 \mu \mathrm{M}$ DMSO, on a RapidCycler 2 instrument (Idaho Technologies, Utah, USA) with the following forward (5'-GGGATTTCGGACGGCTCTCG-3') and reverse (5'-GGCCCAATCCCGCCCAGACTA-3') primers. Melting curves were obtained with a HR-1 High Resolution Melter, and analyzed using the HR-1 Melt Analysis Tool software (Idaho Technologies) and presented as a derivative plot. In each run, samples with a known MDM2 SNP309/SNP285 status (validated by pyrosequencing) were included as reference melting curve. In the presence of a SNP285C allele, no distinction could be made for the SNP309 genotype. The genotype of these samples and samples with indistinct melting curves, were validated by pyrosequencing. A PCR $\left[1 \times\left(94^{\circ} \mathrm{C} / 10 \mathrm{~min}\right) ; 40 \times\left(94^{\circ} \mathrm{C} /\right.\right.$ 30sec, $\left.\left.64^{\circ} \mathrm{C} / 30 \mathrm{sec}, 72^{\circ} \mathrm{C} / 30 \mathrm{sec}\right) ; 1 \times\left(72^{\circ} \mathrm{C} / 10 \mathrm{~min}\right)\right]$ was 
run on a Prime Thermal Cycler (VWR, Leuven, Belgium) using the same primers as described above with the addition of a biotin label on the reverse primer. Pyrosequencing was performed using the PyroMark Gold Q24 reagents kit on a Pyromark Q24 system (Qiagen) with a SNP309 specific primer (5'-CAGGGTAAAGGTCACG-3') or SNP285 specific primer (5'-GGGCTGCGGGGCCGCT-3').

\section{MDM2 mRNA expression levels: quantitative RT-PCR}

MDM2 qRT-PCR was successfully performed on 67 genomic tumor RNA samples and 4 normal lung tissue samples (31 samples were excluded due to insufficient RNA yields or high Ct values). Reverse transcription was performed using the High Capacity RNA-to-cDNA kit (ThermoFisher Scientific) and real-time PCR reaction was performed using the TaqMan Gene Expression Master Mix on a LightCycler480 instrument (Roche, Vilvoorde, Belgium). The hs01066930_m1 (MDM2) TaqMan gene expression assay (ThermoFisher Scientific) was used as target of interest and hs00609296_g1 (HMBS), hs00984230_m1 (B2M), hs01122445_g1 (YWHAZ) were used as housekeeping genes (HKG's) for normalization. These HKG's were selected from a panel of 7 genes tested in 10 representative patient samples (7 NSCLC samples and 3 normal tissue samples). The optimal number and type of HKG's were determined with the geNorm software module of qbasePLUS software (Biogazelle, Zwijnaarde, Belgium). Relative gene expression levels were calculated according to the $\mathrm{Ct}$ method with the qbasePLUS software and plotted against normal lung tissue (SNP309TT/SNP285GG). A second target of interest, for which the protein expression levels were determined in the same samples by IHC, was run simultaneous to assess the reliability of the assay. For this target a significant correlation was demonstrated between mRNA levels and protein expression $(\mathrm{P}<$ $0.001, \mathrm{R} 2=0.497)$. The TP53 status of the tumor was used as positive and negative control. Since MDM2 is a transcriptional target of p53 we expected and observed significant lower MDM2 mRNA expression levels in the presence of mutant p53 (figure 2).

\section{MDM2 protein levels: immunohistochemistry}

MDM2 IHC was successfully performed on 94 samples. Four samples were excluded because the tissue sections did not contain any tumor cells. Five $\mu \mathrm{m}$-thick FFPE tissue sections were prepared and subjected to heat-induced epitope retrieval (HIER) by incubation in a high $\mathrm{pH}$ buffer for $20 \mathrm{~min}$ at $97^{\circ} \mathrm{C}$.
Subsequently, endogenous peroxidase activity was quenched by incubating the slides in peroxidase blocking buffer (DAKO, Heverlee, Belgium) for $5 \mathrm{~min}$. Incubation with the primary monoclonal anti-MDM2 antibody (clone IF2, diluted 1:200 for $30 \mathrm{~min}$, ThermoFisher Scientific) was performed manually at room temperature. Primary incubation was followed by incubation with mouse enhanced polymer-based linker (DAKO) for $30 \mathrm{~min}$ at room temperature. The detection was performed using the Envision FLEX+ detection kit (DAKO). Sections were counterstained with hematoxylin, dehydrated and mounted. Positive controls were included in each staining run and consisted of a MDM2-amplification positive liposarcoma, confirmed by FISH staining. The antibody diluent (DAKO) was used as negative control. Nuclear staining was assessed by two independent observers, including an experienced pathologist, and samples were grouped according to the percentage of MDM2 positive tumor cells $(<5 \%=$ negative; $5-25 \%=$ low; $25-50 \%=$ medium; $>50 \%=$ high MDM2 protein expression, in more than one region). Representative examples of the different types of MDM2 expression are shown in figure 1.

\section{Multiplex Amplification of Specific Targets for Resequencing (MASTR ${ }^{\text {TM}}$ )}

TP53 next generation sequencing was successfully performed on 69 genomic tumor DNA samples using the TP53 MASTR ${ }^{\text {TM }}$ with MID for Illumina Miseq kit (Multiplicom, Niel, Belgium) according to the manufacturer's instructions. A total of 29 samples were excluded because they did not pass the quality control steps of the TP53 MASTRTM kit protocol. Sequencing was performed on a MiSeq system using the MiSeq Reagent Kit v2 (500 cycles) (Illumina, Belgium). Using an in-house annotation and filtering tool, VariantDB, single nucleotide variants were annotated to the TP53 NM_000546 transcript file[12]. SNVs present in the dbSNP137 and 1000 Genomes Project databases were identified as single nucleotide polymorphisms and excluded. The reads were visualised using the Integrative Genomics Viewer software (IGV, version 2.3.67), alligned to the human reference genome (hg19, NCBI build 37). Using the MUT-TP53 2.0 tool, innactivating TP53 mutations were identified [13]. NSCLC cell lines with know TP53 status were included in the run as positive controls (A549 (CCL-185, TP53wt), NCI-1975 (CRL-5908, TP53R273H), NCI-H2228 (CRL-5935, TP53Q331* ) and NCI-H596 (HTB-178, TP53G245C). In each step a non-template control was included. 


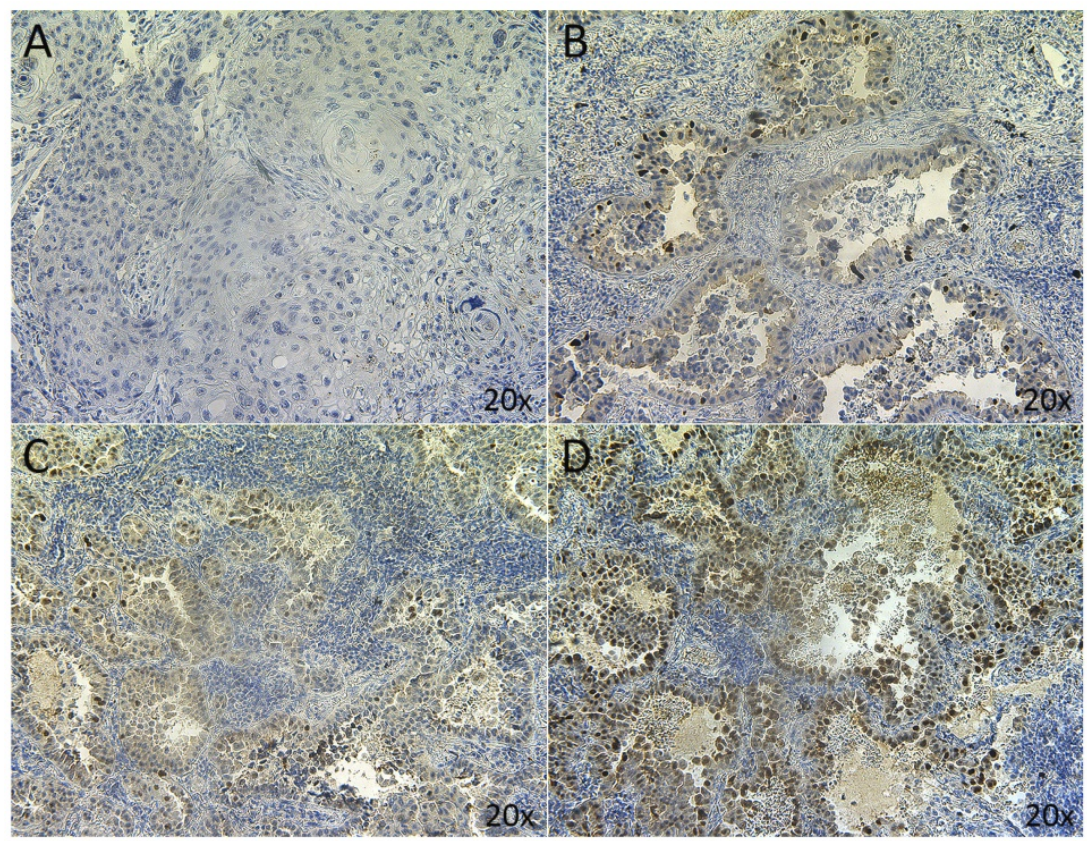

Figure 1. MDM2 nuclear staining in NSCLC. Representative sections of NSCLC tumors classified as (A) <5\%; (B) $5-25 \%$; (C) $25-50 \%$; (D) $>50 \%$.

\section{Statistical analysis}

Associations between the MDM2 SNP309 or SNP285 status and relative MDM2 mRNA expression levels were determined using Student t-test (2 groups) or One-way ANOVA test (> 2 groups).

Associations between SNP309, SNP285, MDM2 protein expression and clinicopathological characteristics of NSCLC patients were investigated by $\chi^{2}$ analysis or Fisher's exact test (when appropriate) for categorical variables and the Student t-test or One-way ANOVA analysis for continuous variables.

Prognostic value was assessed by survival analysis. PFS was indicated as the time until disease progression occurred. OS was indicated as the time until cancer related death occurred. Univariate OS or PFS probability was estimated using the Kaplan-Meier method. Statistical significance was determined using the log-rank test. In addition to univariate analysis, a multivariate cox proportional-hazard model was fitted to identify independent prognostic markers, presented as a hazard ratio (HR) and its 95\% confidence interval (CI). All analyses were performed using SPSS version 23 and significance was reached if $p<0.05$ (two-tailed).

\section{Results}

\section{SNP309/SNP285 distribution and association with clinicopathological data}

In this study 98 NSCLC adenocarcinoma patients were analyzed for MDM2 SNP309 and SNP285 status. MDM2 SNP309 and SNP285 frequencies, MDM2 IHC scoring and TP53 status are presented in table 1.
Table 1. Patient characteristics for MDM2 and TP53

\begin{tabular}{lll}
\hline & No. & $(\mathbf{0})$ \\
\hline MDM2 SNP309 & 39 & $(39.8)$ \\
TT & 38 & $(38.8)$ \\
GT & 21 & $(21.4)$ \\
GG & & \\
MDM2 SNP285 & 91 & $(92.9)$ \\
GG & 7 & $(7.1)$ \\
GC & 0 & $(0.0)$ \\
CC & & \\
MDM2 IHC (n=94) & 60 & $(61.2)$ \\
$<5 \%$ & 25 & $(25.5)$ \\
$5-25 \%$ & 6 & $(6.1)$ \\
$25-50 \%$ & 3 & $(3.1)$ \\
$>50 \%$ & & \\
TP53 status (n=69)* & 40 & $(58.0)$ \\
Wild type & 29 & $(42.0)$ \\
Mutant &
\end{tabular}

*Mutant: inactivating TP53 mutations (activity determined using the MUT-TP53 2.0 tool)

The SNP309G allele was frequently present in $60.2 \%$ of the patients. The SNP285C allele was only present as a heterozygous genotype G/C in 7 patients $(7.1 \%)$ and in the presence of the SNP309G allele. TP53 inactivating mutations occurred in 29 patients $(42 \%)$ and were restricted to exons 4-8.

Table 2 summarizes the frequencies of the different genotypes according to the patients' clinicopathological data. The SNP309G allele was more frequently present in wild type TP53 tumors ( $p$ $=0.047$ ). No associations were found for SNP309 and tumor stage, T-classification, differentiation status, metastasis or smoking status. 
Table 2. Association MDM2 SNP309 and SNP285 with clinicopathological characteristics

\begin{tabular}{|c|c|c|c|c|c|c|c|c|c|}
\hline & \multicolumn{6}{|c|}{ MDM2 SNP309 } & \multicolumn{3}{|c|}{ MDM2 SNP285 } \\
\hline & TT (\%) & GT (\%) & GG (\%) & $\mathbf{P}$ & GT/GG (\%) & $\mathbf{P}^{* * *}$ & GG (\%) & GC(\%) & $\mathbf{P}$ \\
\hline Median Age \pm StDev & $64.2 \pm 9.2$ & $64.2 \pm 9.5$ & $64.7 \pm 10.6$ & 0.980 & $64.3 \pm 9.8$ & 0.957 & $65.0 \pm 9.2$ & $55.1 \pm 10.0$ & 0.008 \\
\hline \multicolumn{10}{|l|}{ Gender } \\
\hline Male & $22(56.4)$ & $26(68.4)$ & $16(76.2)$ & \multirow[t]{2}{*}{0.269} & $42(71.2)$ & \multirow[t]{2}{*}{0.193} & $59(64.8)$ & $5(71.4)$ & \multirow[t]{2}{*}{0.538} \\
\hline Female & $17(43.6)$ & $12(31.6)$ & $5(23.8)$ & & $17(28.8)$ & & $32(35.2)$ & $2(28.6)$ & \\
\hline \multicolumn{10}{|l|}{ Stage* } \\
\hline I & $18(46.2)$ & $18(47.4)$ & $7(33.3)$ & \multirow[t]{4}{*}{0.650} & $25(42.4)$ & \multirow[t]{4}{*}{0.471} & $41(45.1)$ & $2(28.6)$ & \multirow[t]{4}{*}{0.140} \\
\hline II & $12(30.8)$ & $8(21.1)$ & $6(28.6)$ & & $14(23.7)$ & & $24(26.4)$ & $2(28.6)$ & \\
\hline III & $8(20.5)$ & $9(23.7)$ & $5(23.8)$ & & $14(23.7)$ & & $21(23.1)$ & $1(14.3)$ & \\
\hline IV & $1(2.6)$ & $3(7.9)$ & $3(14.3)$ & & $6(10.2)$ & & $5(5.5)$ & $2(28.6)$ & \\
\hline \multicolumn{10}{|l|}{ T-classification* } \\
\hline $\mathrm{T} 1$ & $12(30.8)$ & $15(39.5)$ & $5(23.8)$ & \multirow[t]{4}{*}{0.281} & $20(33.9)$ & \multirow[t]{4}{*}{0.952} & $32(35.2)$ & $0(0.0)$ & \multirow[t]{4}{*}{0.026} \\
\hline $\mathrm{T} 2$ & $15(38.5)$ & $11(28.9)$ & $12(57.1)$ & & $23(39.0)$ & & $32(35.2)$ & $6(85.7)$ & \\
\hline T3 & $9(23.1)$ & $11(28.9)$ & $2(9.5)$ & & $13(22.0)$ & & $22(24.2)$ & $0(0.0)$ & \\
\hline $\mathrm{T} 4$ & $3(7.7)$ & $1(2.6)$ & $2(9.5)$ & & $3(5.1)$ & & $5(5.5)$ & $1(14.3)$ & \\
\hline \multicolumn{10}{|l|}{ Differentation* } \\
\hline Poor & $10(25.6)$ & $11(28.9)$ & 7 (33.3) & \multirow[t]{3}{*}{0.915} & $18(30.5)$ & & $25(27.5)$ & $3(42.9)$ & \\
\hline Moderate & $14(35.9)$ & $12(31.6)$ & $8(38.1)$ & & $20(33.9)$ & \multirow[t]{2}{*}{0.872} & $32(35.2)$ & $2(28.6)$ & \\
\hline Strong & $15(38.5)$ & $15(39.5)$ & $6(28.6)$ & & $21(35.6)$ & & $34(37.4)$ & $2(28.6)$ & \\
\hline \multicolumn{10}{|l|}{ Metastasis** } \\
\hline Yes & $9(23.1)$ & 12 (31.6) & $6(28.6)$ & \multirow[t]{2}{*}{0.701} & $18(30.5)$ & \multirow[t]{2}{*}{0.493} & $22(24.2)$ & $5(71.4)$ & \multirow[t]{2}{*}{0.016} \\
\hline No & $30(76.9)$ & $26(68.4)$ & $15(71.4)$ & & $41(69.5)$ & & $69(75.8)$ & $2(28.6)$ & \\
\hline \multicolumn{10}{|l|}{ Smoking Status $(n=85)$} \\
\hline Smoker & $30(83.3)$ & $29(90.6)$ & $17(100.0)$ & \multirow[t]{2}{*}{0.177} & $46(93.9)$ & \multirow[t]{2}{*}{0.159} & $72(90.0)$ & $4(80.0)$ & \multirow[t]{2}{*}{0.437} \\
\hline Never-smoker & $6(16.7)$ & $3(9.4)$ & $0(0.0)$ & & $3(6.1)$ & & $8(10.0)$ & $1(20.0)$ & \\
\hline \multicolumn{10}{|l|}{ MDM2 IHC (n=94) } \\
\hline$<5 \%$ & $29(74.4)$ & $20(55.6)$ & $11(57.9)$ & \multirow[t]{4}{*}{0.188} & $31(56.4)$ & 0.068 & $56(63.6)$ & $4(66.7)$ & 0.866 \\
\hline $5-25 \%$ & $8(20.5)$ & $12(33.3)$ & $5(26.3)$ & & $17(30.9)$ & & $23(26.1)$ & $2(33.3)$ & \\
\hline $25-50 \%$ & $0(0.0)$ & $3(8.3)$ & $3(15.8)$ & & $6(10.9)$ & & $6(6.8)$ & $0(0.0)$ & \\
\hline$>50 \%$ & $2(5.1)$ & $1(2.8)$ & $0(0.0)$ & & $1(1.8)$ & & $3(3.4)$ & $0(0.0)$ & \\
\hline TP53 Status $(n=69)$ & & & & & & & & & \\
\hline Wild Type & $11(40.7)$ & $18(62.1)$ & $10(76.9)$ & 0.071 & $28(66.7)$ & 0.047 & $36(55.4)$ & $3(75.0)$ & 0.442 \\
\hline Mutant & $16(59.3)$ & $1137.9)$ & $2(23.1)$ & & $14(33.3)$ & & $20(44.6)$ & $1(25.0)$ & \\
\hline
\end{tabular}

* At time of diagnosis ** Initially and/or after progression *** TT vs. GT/GG

The SNP285GC genotype was significantly associated with $\mathrm{T} 2$ primary tumor classification status $(p=0.026)$ and tumor metastasis $(p=0.016)$, but not with gender, tumor stage, differentiation, smoking status or TP53 mutational status. The median age at diagnosis was significantly lower in patients harboring the SNP285GC genotype $(p=0.008)$.

\section{Association of SNP309/285 with MDM2 mRNA and MDM2 protein expression levels}

Relative MDM2 mRNA expression levels were successfully obtained from 67 tumor samples. No difference in mRNA expression was observed according to SNP309 status (figure 2). However, in the presence of SNP285GC genotype, MDM2 mRNA levels were significantly increased compared to the SNP285GG genotype $(p=0.025)$. No significant association was observed between MDM2 protein expression and mRNA levels $(\mathrm{p}=0.922$, data not shown). A strong trend for an association between the SNP309G allele and increased MDM2 protein expression was observed ( $\mathrm{p}=0.068$; table 2$)$. No association was observed for SNP285 and MDM2 protein levels ( $p=0.866$; table 2$)$. Representative IHC samples are shown in figure 1 . It should be mentioned that MDM2 protein levels were very heterogeneous within each sample. Since MDM2 is a transcriptional target of p53, the presence of mutant p53 could possibly affect MDM2 mRNA levels. Indeed, we observed significantly lower MDM2 mRNA levels in the presence of inactivating TP53 mutations ( $\mathrm{p}<$ 0.0001 , figure 2).

\section{The prognostic value of MDM2 SNP309/SNP285 according to TP53 status}

Follow-up OS and PFS data was available for all 98 patients. At the end of the observation period, 41 $(41.8 \%)$ patients were deceased and 34 (34.7\%) showed disease progression.

The Kaplan-Meier method was used to assess OS and PFS probability for MDM2 SNP309 and SNP285 status. In the TP53 wild type group, the SNP309G allele was clearly associated with worse OS (figure 3). Since no event (death) occurred in the TT-genotype group, log-rank testing could not be performed. However, the presence of the SNP309G allele was significantly associated with death $(\mathrm{p}=0.038)$. This effect was not observed in the TP53 mutant group $(\mathrm{p}=$ 0.438). Grouping by TP53 status did not affect PFS. 

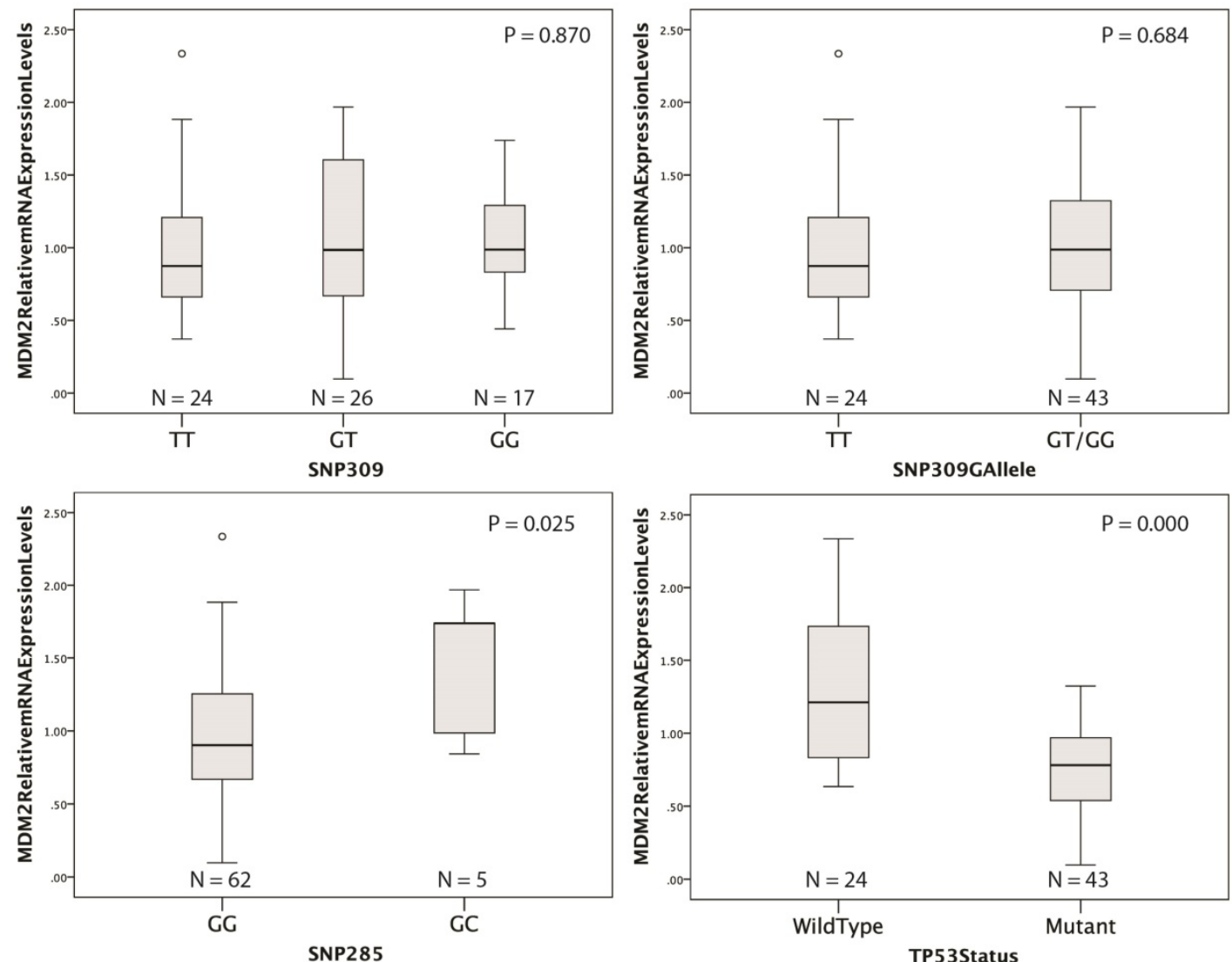

Figure 2. Distribution of relative MDM2 mRNA expression levels according to the SNP309, SNP285 or TP53 mutational status. P $<0.05$ indicates statistical significance.
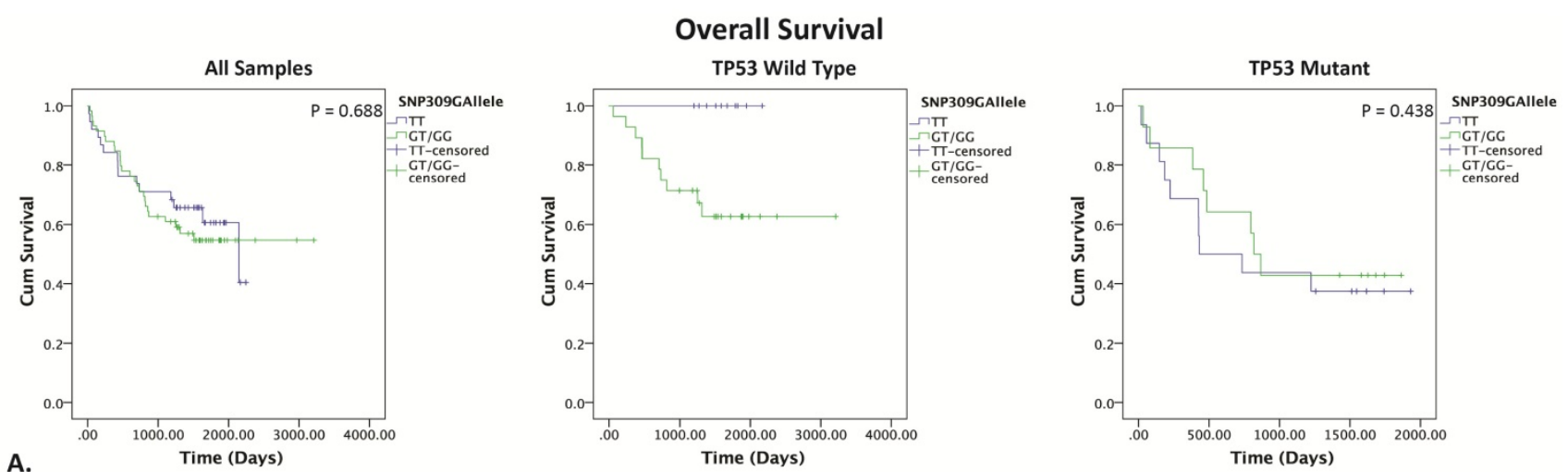

A.
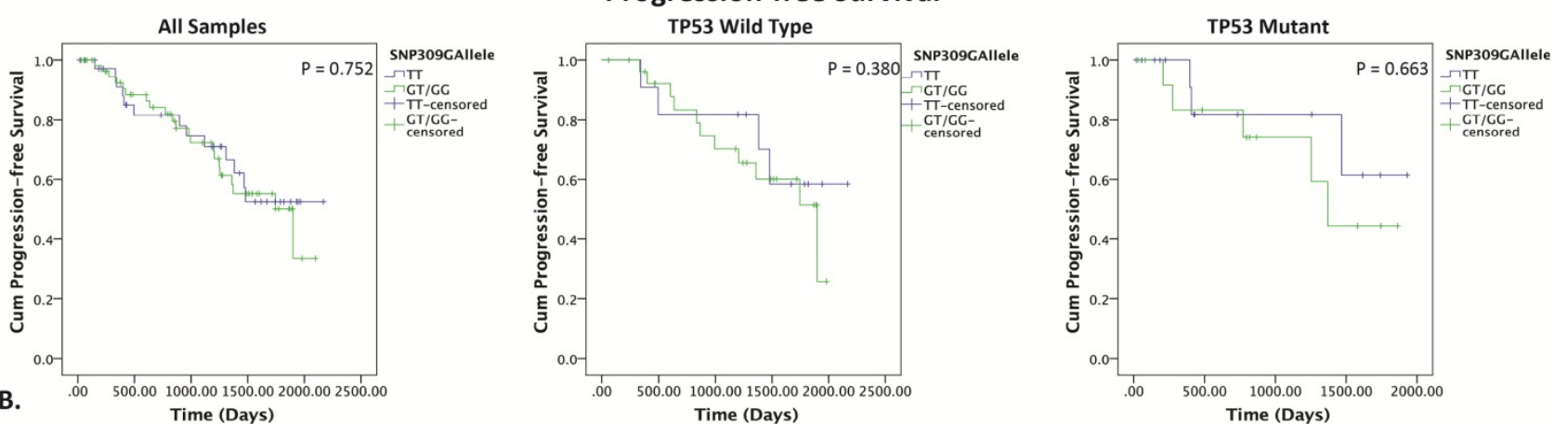

Figure 3. Kaplan-Meier survival curves for SNP309 grouped by TP53 status. (A) Kaplan-Meier survival curves for overall survival, SNP309TT (blue) vs. SNP309GT/GG (green). (B) Kaplan-Meier survival curves for progression free survival, SNP309TT (blue) vs. SNP309GT/GG (green). P<0.05 indicates statistical significance determine by the log rank test. 
The SNP285GC genotype did not affect OS $(p=$ 0.922 ), but was strongly associated with a significantly worse PFS as shown in figure $4(p=0.002)$. Patients were not stratified according to TP53 status due to low sample sizes.

In addition to univariate analysis, we fitted a Cox proportional hazard model with SNP309 and SNP285 status as predictor for OS and accounting for potential confounders including gender, age, differentiation, T-classification, metastasis and tumor stage (table 3). As expected, neither SNP309 nor SNP285 acted as an independent prognostic marker for OS in the overall patient group. As stated previously, outcome could not be grouped according to TP53 status in uni- and multivariate analysis.

Similarly, a Cox model was fitted for SNP309 and SNP285 status as predictor for PFS, accounting for gender, age, differentiation, T-classification and tumor stage (table 3).

Stepwise backward model building showed that SNP285 acted as a significant predictor for adverse PFS, accounting for age $(\mathrm{HR}=3.97 ; 95 \% \mathrm{CI}: 1.51-$ 10.42; $\mathrm{p}=0.005)$.

Table 3. Independent predictors of OS and PFS (Multivariate Cox regression model)

\begin{tabular}{lll}
\hline Overall Survival & & \\
\hline Predictor & HR $(95 \%$ CI $)$ & P-value \\
Tumor Stage & $1.69(1.25-2.28)$ & 0.001 \\
Progression-free Survival & \\
Predictor & HR $(95 \%$ CI) & P-value \\
SNP285 (GC vs GG) & $3.97(1.51-10.42)$ & 0.005 \\
Age $(>65$ vs. $\leq 65)$ & $0.53(0.26-1.08)$ & 0.08
\end{tabular}

Factors included in the backward conditional model: SNP309, SNP285, gender, age, differentiation, T-classification, tumor stage and metastasis (in OS model only). HR: Hazard Ratio; CI: confidence interval; $p<0.05$ indicates statistical significance.

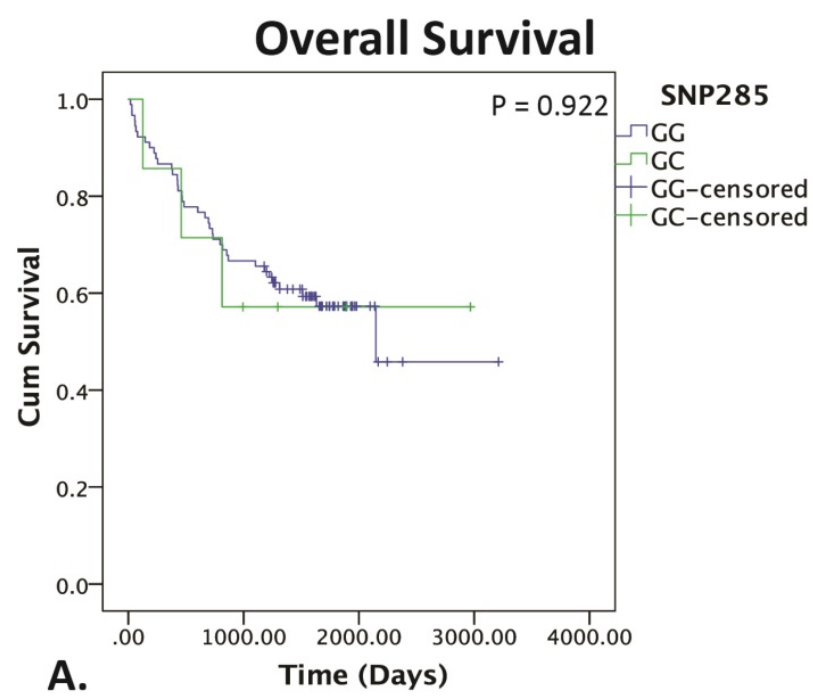

\section{Discussion}

The present study shows that the MDM2 SNP309G allele was not associated with increased MDM2 mRNA levels, in contrast to the MDM2 SNP285C allele, which significantly increased relative MDM2 mRNA expression. This seems inconsistent with the theory that the SNP285C allele acts as an antagonist to the SNP309G allele and reduces MDM2 expression by overriding its effect on the binding of the Sp1 transcription factor to the MDM2 promoter [5]. However, previous studies did not determine actual MDM2 transcript levels showing the effect of the SNP285C allele on MDM2 mRNA expression levels. In the past, only one other study determined MDM2 mRNA levels in lung cancer, and showed higher mRNA levels in tumors with the SNP309G allele, particularly in wild type p53 tumors, showing a stronger effect compared to our present study [14]. In their study, mRNA was isolated from frozen tumor sections, resulting in mRNA of higher quality compared to that isolated from FFPE tissue, which was a limiting factor in this study. However, we did compensate for the possible lower mRNA quality by using a large panel of stable housekeeping genes, which can compensate for inter-sample differences in RNA quality. Since p53 is an important regulator of MDM2 transcription, we expected reduced MDM2 transcription levels in the TP53 mutant subgroup, what indeed was confirmed by our data. The SNP309TG/GG genotype showed a strong trend towards increased MDM2 protein levels compared to the SNP309TT genotype, indicating an effect of SNP309 on MDM2 protein levels as previously suggested.

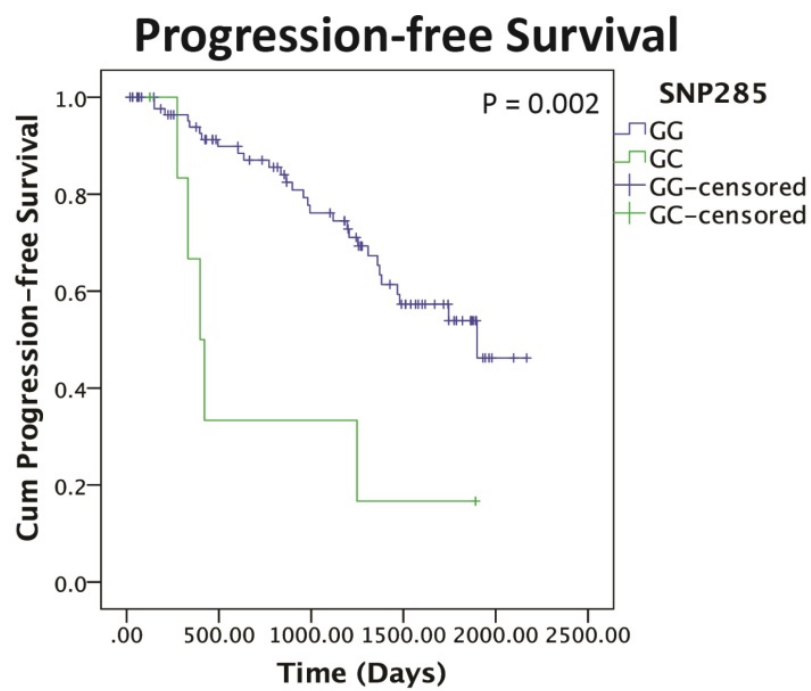

Figure 4. Kaplan-Meier survival curves for SNP285. Kaplan-Meier survival curve for OS (left) and PFS (right) of SNP285GG (blue) vs. SNP285GC (green). $P<0.05$ indicates statistical significance determine by the log rank test. 
MDM2 protein expression was very heterogeneous within the samples, which could account for the lack of association between of MDM2 mRNA levels and MDM2 protein expression levels. MDM2 mRNA was isolated from 5-10 FFPE tissue slides in comparison to one slide for MDM2 IHC analysis, consequently giving a better representation of the whole tumor. Since MDM2 was not homogenously expressed in all tumor cells harboring the SNP309G allele, it seems that presence of this SNP does not lead to constitutive overexpression of MDM2.

The main goal of this study was to determine the prognostic value of SNP309 and SNP285 status in NSCLC patients, since contradictory results have been reported previously [4]. We showed that the SNP309TG/GG genotype resulted in worse OS compared to the SNP309TT genotype in the TP53 wild type patient group. The significant association of the SNP309G allele and the presence of wild type TP53 further supports the theory that the SNP309G allele has a more prominent role in wild type p53 tumors, possibly by increased inhibition of wild type p53's function due to increased MDM2 protein levels. Only one previous study took the p53 status into account, limited to stage I tumors, showing a better OS in wild type p53 patients harboring the SNP309TG or SNP309GG genotype [15]. Recently, Enokida et al. reported similar results, showing that the SNP309TT genotype was predictive for poorer survival in stage I patients, without taking the p53 status into account [16]. These data seem inconsistent with our results in stage 1 tumors (data not shown). However, the difference in ethnicity between these studies (Asian) and ours (Caucasian) has to be taken into account, and could account for the difference in prognostic value. Han et al. previously showed the SNP309TG/GG genotype was associated with worse OS and PFS in late stage tumors, consistent with our results in TP53 wild type patients [17].

To the best of our knowledge we are the first to show the significant association of SNP285C allele with a lower age at diagnosis and a worse PFS, which could be accounted for by the significant association with metastasis. These findings, in addition to the increased MDM2 transcript levels, suggest that the SNP285C allele might be a valuable negative prognostic marker for NSCLC patients. Only one study reported the prognostic value of SNP285 and showed that the SNP285C allele did not antagonize the effect of the presence of the SNP309G allele on lung cancer risk or survival, but the effect of SNP285 by itself or the relation with PFS was not determined in this study [9]. Since the GC genotype was only present $7.1 \%$ of all our patients, this hypothesis is based on a limited sample size. Therefore, larger patient groups could further support our findings.

In conclusion, we showed that the prognostic value of SNP309 on overall survival is limited to p53 wild type patients and that the SNP285C allele is associated with increased MDM2 transcription and metastasis and acts as a strong predictor for worse PFS. Our data supports the prognostic value of both SNP309 and SNP285 polymorphisms in NSCLC patients, providing a strong rational for the use of MDM2 inhibitors for the treatment of NSCLC in the presence of wild type p53, either or not in combination with conventional therapies [18].

\section{Acknowledgements}

C. Deben and J. Jacobs were funded by the Agency for Innovation by Science and Technology, Flanders [grant numbers 111063, 120822]. A. Wouters is funded by Research Foundation Flanders [grant number $1297813 \mathrm{~N}]$. The authors would also like to thank Multiplicom for funding the TP53 MASTR ${ }^{\text {TM }}$ kit and technical assistance.

\section{Competing Interests}

The authors have declared that no competing interest exists.

\section{References}

1. Meek DW, Hupp TR. The regulation of MDM2 by multisite phosphorylation--opportunities for molecular-based intervention to target tumours? Seminars in cancer biology. 2010; 20: 19-28.

2. Moll UM, Petrenko O. The MDM2-p53 interaction. Molecular cancer research : MCR. 2003; 1: 1001-8.

3. Bond GL, Hu W, Bond EE, Robins H, Lutzker SG, Arva NC, et al. A single nucleotide polymorphism in the MDM2 promoter attenuates the p53 tumor suppressor pathway and accelerates tumor formation in humans. Cell. 2004; 119: 591-602.

4. Deben C, Deschoolmeester V, Lardon F, Rolfo C, Pauwels P. TP53 and MDM2 genetic alterations in non-small cell lung cancer: Evaluating their prognostic and predictive value. Critical reviews in oncology/hematology. 2016; 99: 63-73.

5. Knappskog S, Bjornslett M, Myklebust LM, Huijts PE, Vreeswijk MP, Edvardsen $\mathrm{H}$, et al. The MDM2 promoter SNP285C/309G haplotype diminishes Sp1 transcription factor binding and reduces risk for breast and ovarian cancer in Caucasians. Cancer cell. 2011; 19: 273-82.

6. Knappskog S, Gansmo LB, Dibirova K, Metspalu A, Cybulski C, Peterlongo P, et al. Population distribution and ancestry of the cancer protective MDM2 SNP285 (rs117039649). Oncotarget. 2014; 5: 8223-34.

7. Paulin FE, O'Neill M, McGregor G, Cassidy A, Ashfield A, Ali CW, et al. MDM2 SNP309 is associated with high grade node positive breast tumours and is in linkage disequilibrium with a novel MDM2 intron 1 polymorphism. BMC cancer. 2008; 8: 1-10.

8. Gansmo LB, Knappskog S, Romundstad P, Hveem K, Vatten L, Lonning PE. Influence of MDM2 SNP309 and SNP285 status on the risk of cancer in the breast, prostate, lung and colon. International journal of cancer. 2015; 137: 96-103.

9. Ryan BM, Calhoun KM, Pine SR, Bowman ED, Robles AI, Ambs S, et al. MDM2 SNP285 does not antagonize the effect of SNP309 in lung cancer. International journal of cancer. 2012; 131: 2710-6.

10. Knappskog S, Trovik J, Marcickiewicz J, Tingulstad S, Staff AC, MoMa TECsg, et al. SNP285C modulates oestrogen receptor/Sp1 binding to the MDM2 promoter and reduces the risk of endometrial but not prostatic cancer. European journal of cancer. 2012; 48: 1988-96.

11. Deben C, Zwaenepoel K, Boeckx C, Wouters A, Pauwels P, Peeters M, et al. Expression analysis on archival material revisited: isolation and quantification of RNA extracted from FFPE samples. Diagnostic molecular pathology : the American journal of surgical pathology, part B. 2013; 22: 59-64. 
12. Vandeweyer G, Van Laer L, Loeys B, Van den Bulcke T, Kooy RF. VariantDB: a flexible annotation and filtering portal for next generation sequencing data. Genome Med. 2014; 6: 74.

13. Soussi T, Hamroun D, Hjortsberg L, Rubio-Nevado JM, Fournier JL, Beroud C. MUT-TP53 2.0: a novel versatile matrix for statistical analysis of TP53 mutations in human cancer. Hum Mutat. 2010; 31: 1020-5.

14. Chien WP, Wong RH, Cheng YW, Chen $\mathrm{CY}$, Lee H. Associations of MDM2 SNP309, transcriptional activity, mRNA expression, and survival in stage I non-small-cell lung cancer patients with wild-type p53 tumors. Ann Surg Oncol. 2010; 17: 1194-202.

15. Chien WP, Wong RH, Wu TC, Cheng YW, Chen CY, Lee H. Potential increase in the prognostic value of p53 mutation by Pro72 allele in stage I non-small-cell lung cancer. Ann Surg Oncol. 2009; 16: 1918-24.

16. Enokida Y, Shimizu K, Atsumi J, Kakegawa S, Takase Y, Kaira K, et al. Prognostic potential of the MDM2 309T>G polymorphism in stage I lung adenocarcinoma. Cancer Med. 2016; 5: 1791-801.

17. Han JY, Lee GK, Jang DH, Lee SY, Lee JS. Association of p53 codon 72 polymorphism and MDM2 SNP309 with clinical outcome of advanced nonsmall cell lung cancer. Cancer. 2008; 113: 799-807.

18. Deben C, Wouters A, Op de Beeck K, van Den Bossche J, Jacobs J, Zwaenepoel

$\mathrm{K}$, et al. The MDM2-inhibitor Nutlin-3 synergizes with cisplatin to induce p53 dependent tumor cell apoptosis in non-small cell lung cancer. Oncotarget. 2015; 6: 22666-79. 